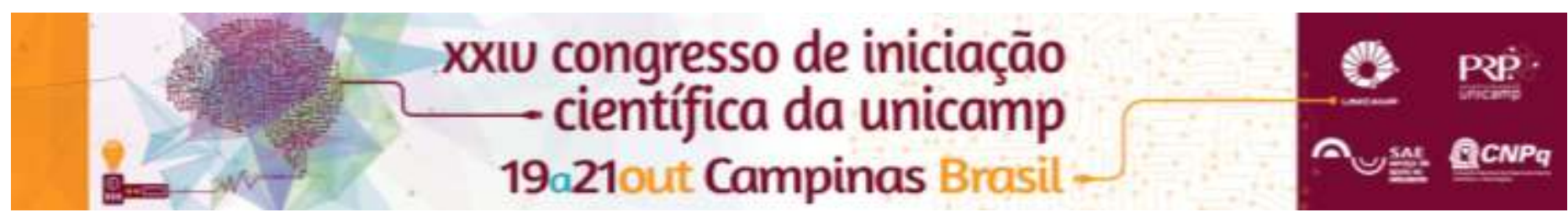

\title{
EFFECTS OF P-MAPA ASSOCIATED WITH GEMCITABINE IN THE PANCREATIC CANCER: HISTOLOGIC EFFECTS
}

\author{
Maísa M. Pereira*, Mariana M. dos Santos, Iseu N. da Silva, Nelson Durán, Wagner J. Fávaro, Patrick V. Garcia.
}

\begin{abstract}
Currently antitumor therapies consist in the neoplasic cells attack by chemotherapy and radiotherapy. Successful treatment depends on the type of cancer, because each cancer has particular characteristics of survival and immune evasion. The neoplasic cell biology studies demonstrated a persistent immunosuppressive environment surrounding transformed cells. Thus, compounds that are capable of acting as agonists of Toll-like receptors (TLRs) may represent promising candidates to be developed as medicaments against cancer. Thus, the general objectives of this study are to characterize and compare the histologic effects of P-MAPA associated with the chemotherapeutic agent Gemcitabine to treat pancreatic cancer in rats.
\end{abstract}

\section{Key words:}

Pancreatic cancer, immunotherapy, histopathology.

\section{Introduction}

The pancreatic cancer is one of the most aggressive tumors, being responsible for $5 \%$ of deaths by cancer worldwide. Strong evidences suggest that the main oncogenic pathways are adapted to the metabolism of the tumor to guarantee its progression and development. In this context, P-MAPA imunomodulator should be considered a new perspective to fight some types of cancer, including the pancreatic cancer. Objectives: The objectives of this study are to characterize and to compare the histopathological effects of the P-MAPA immunotherapy in association to chemotherapy with gemcitabine for the treatment of induced pancreatic cancer in rats. Methodology: To induce pancreatic cancer 7,12Dimethylbenz[a]anthracene (DMBA) crystals were implanted in the head of the pancreas of 40 male Fischer 344 rats. Ten other animals did not received DMBA and were considered as controls. After 120 days of induction, all animals were submitted to ultrasonography to confirm the existence of the pancreatic cancer and were divided in 5 groups (10 animals each): Control group (G1): received intraperitoneal injections of $5 \mathrm{ml} / \mathrm{kg}$ of $0.9 \%$ saline three times a week for 6 consecutive weeks; Cancer group (G2): received the same treatment as G1; Cancer + P-MAPA group (G3): received intraperitoneal injections of $5 \mathrm{mg} / \mathrm{kg}$ of P-MAPA (Farmabrasilis, São Paulo, Brazil), three times a week for 6 consecutive weeks; Cancer + Gemcitabine group (G4): received intraperitoneal injections of $10 \mathrm{mg} / \mathrm{kg}$ Gemcitabine once weekly for 6 consecutive weeks (modified Kim et al, 2014); Cancer + Gemcitabine + PMAPA group (G5): received simultaneous treatment with Gemcitabine and P-MAPA according to the same protocols established in G3 and G4. After 30 days of treatment, pancreatic samples were collected and submitted to analysis

\section{Results and Discussion}

Histopathology results show the effectiveness of the experimental model to pancreatic cancer by DMBA in the rats as well as a progressive tissue repair in therapeutic methods, particularly in the Cancer + P-MAPA group (G3).
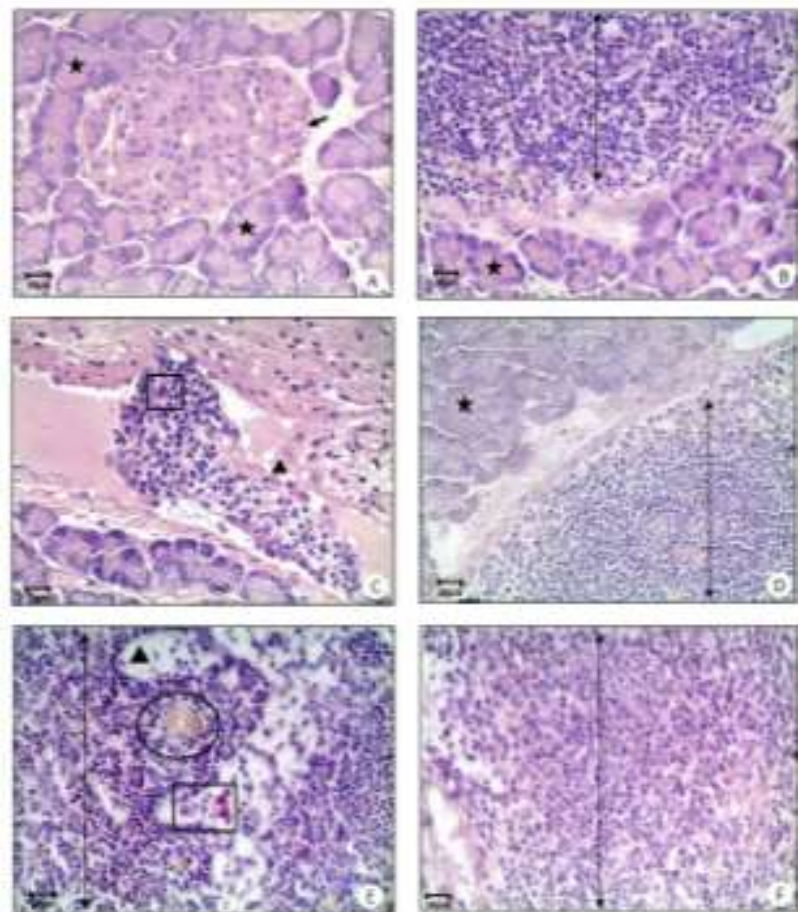

Figure 1A - 1F: Photomicrographs of pancreas of male rats. G1 (A) G2 $(B-C), G 3(D), G 4(E)$ and $G 5(F)$. In (A) observed normal acinar cells (stars) representing the exocrine region (thick arrow), characterized endocrine gland region. In (B) we can observe the presence of infiltrate in the acinar cells (longitudinal arrow). In (C) is observed vacuolar formations in atypical acinar cells, featuring cystic adenocarcinoma (head of thick arrow), and the presence of macrophage (square). In (D) observed the presence of infiltrate (longitudinal arrow) and normal acinar cells (star). It is noted in (E) atrophic glands (circle), macrophages (square) and neoplastic acini (longitudinal arrow). It can be seen in $(F)$ a cell infiltrate (longitudinal arrow). Coloration: Hematoxylin and Eosin (HE). Scale: $50 \mu \mathrm{m}, 400 \mathrm{x}$ increase.

\section{Conclusions}

The reported results present a new therapeutic perspective and new studies to its viability.

\section{Acknowledgement}

Sponsor Institution: São Paulo Research Foundation FAPESP. 